\title{
Beam Species Characteristics of High Current Ion Source for EAST Neutral Beam Injector
}

\author{
Y Wang ${ }^{1,2}$, Z M Liu ${ }^{1,2}$, J Y Yan ${ }^{1,2}$, L Z Liang ${ }^{1} \&$ C D Hü ${ }^{1,2}$ \\ ${ }^{1}$ Institute of Plasma Physics, Chinese Academy of Sciences, Hefei, Anhui, 230031, China \\ ${ }^{2}$ University of Science and Technology of China, Hefei, Anhui, 230026, China \\ Correspondence: L Z Liang, Institute of Plasma Physics, Chinese Academy of Sciences, Hefei, Anhui, 230031, \\ China. E-mail: lzliang@ipp.ac.cn
}

\author{
Received: April 15, 2016 Accepted: May 4, 2016 Online Published: May 17, 2016 \\ doi:10.5539/apr.v8n3p104 URL: http://dx.doi.org/10.5539/apr.v8n3p104
}

\begin{abstract}
During the experiment of high-current ion source on EAST NBI test bed, the Doppler shift spectroscopy (DSS) is used to measure the hydrogen alpha lines emitted by excited atoms. The multi-peak Gaussian fitting gives the distribution of the original DSS spectra data, the beam energy and the fractions of the beam species can be calculated for different operation parameters of ion source. It is known that the proton ratio increases with the arc power, the beam energy and the perveance, this phenomenon can provide a reference for higher power neutral beam extraction. According to the experimental results, the maximum proton ratio is near $78 \%$ under the arc power is about $65 \mathrm{~kW}$. This result indicates that the high-current ion source extraction match the design requirements.
\end{abstract}

Keywords: neutral beam injector, Doppler shift spectroscopy, beam species

\section{Introduction}

Neutral beam injection (NBI) has widely been used in thermal nuclear fusion experiments for auxiliary heating (Hu \& Team, 2012; Jeong et al., 2012; Liang, Hu, Xie, Guo, \& Xie, 2011; Thomas, Grierson, Burgos, \& Van Zeeland, 2012; Tsumori et al., 2006). The high proton ratio can improve the efficiency of plasma heating and current drive, so it is essential to know just the variations of the beam species with discharge parameters. The beam species measurements have been taken for the high-current ion source conditioning on the EAST NBI test bed, and the experimental results will be described in the paper.

For the EAST NBI test bed experiment, the design value of neutral beam energy is from $50 \mathrm{keV}$ to $80 \mathrm{keV}(\mathrm{Hu}$, Xie, \& Team, 2012). The conventional calorimetric and electrical measuring devices suffer severe damages from high power neutral beams (Kamperschroer et al., 1987). To diagnose the performance of extracting beams from EAST-NBI ion source, we develop a non-contact DSS optical diagnostic system by detecting the hydrogen Balmer-alpha emission lines. It can give the beam components by the intensity of spectral lines and effective cross sections. The DSS system can achieve online and real time measurement, as well as the computerized data restore and the storage capability allow a further data analysis (Burrell, Cooper, Smith, \& Steele, 1980). The paper focuses on the variation of the beam species with discharge parameters, such as the arc power, the beam energy, the perveance and the gas flow rate, according to the data acquired from the DSS optical diagnostic system.

\section{Experimental Setup}

As shown in Figure 1 (a), the EAST NBI system mainly consists of ion source, neutralizer cell, vacuum chamber, bending magnet, residual ion dump, calorimeter, control system, cooling water system, diagnostic system. Among these, the ion source is the key element, comprising a magnetic cusp bucket plasma generator and a multi-slit tetrode accelerator. Deuterium gas is puffed into the arc plasma generator and ionized, and then the positive ions $\left(\mathrm{D}^{+}, \mathrm{D}_{2}{ }^{+}\right.$and $\left.\mathrm{D}_{3}{ }^{+}\right)$are extracted and accelerated through an electrode system in the accelerator to form ion beam. Part of the ion beam convert to the neutral beam through charge exchange collisions with the gas target in the neutralizer cell. After that, the residual ions are bent into ion dump by the bending magnet component. And the energetic neutral beam move further downstream to the calorimeter for testing or into the EAST for heating. The EAST NBI test bed has a similar design with the EAST NBI system. 
The experiment has developed the Doppler shift spectroscopy optical system to diagnose the beam components. Since the electron-atom/ion collisions and the background gas in the neutralizer cell have a great impact on the results (Liang, Hu, Xie, Xie, \& Nbi-Team, 2010; Uhlemann, Hemsworth, Wang, \& Euringer, 1993), and the large deviation will appear if the following calculation model is used in the neutralizer. So we measure the Ha lines which are collected between neutralizer and bending magnet in this experiment. We have set up a lens to collect the hydrogen alpha lines in the horizontal plane. The viewing angle, by definition, is an angle between sightline and the beam axis. The sightline makes an angle of $113^{\circ}$ with the beam line near the bending magnet as shown in Figure 1 (b). The optical fiber transmits the optical signals to a Czerny-Turner spectrometer with equipping a charge coupled device (CCD) camera. The CCD camera converts optical signals to electrical signals, the experimental data are recorded in the computer finally.

(a)

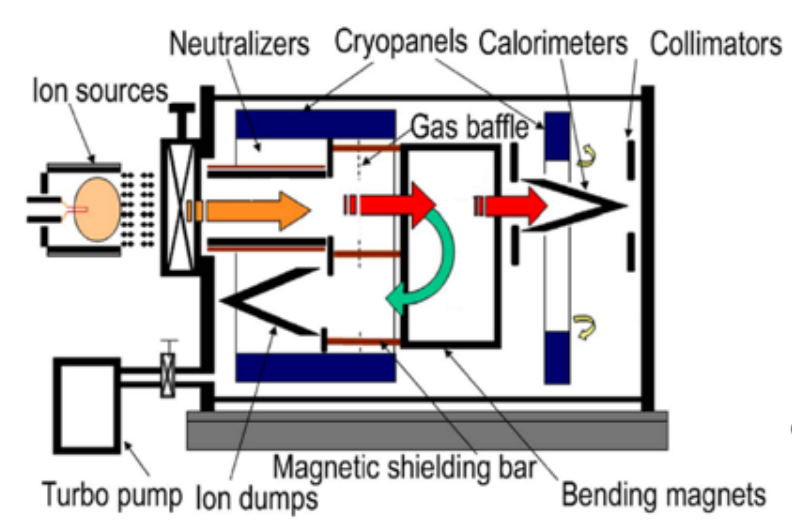

(b)

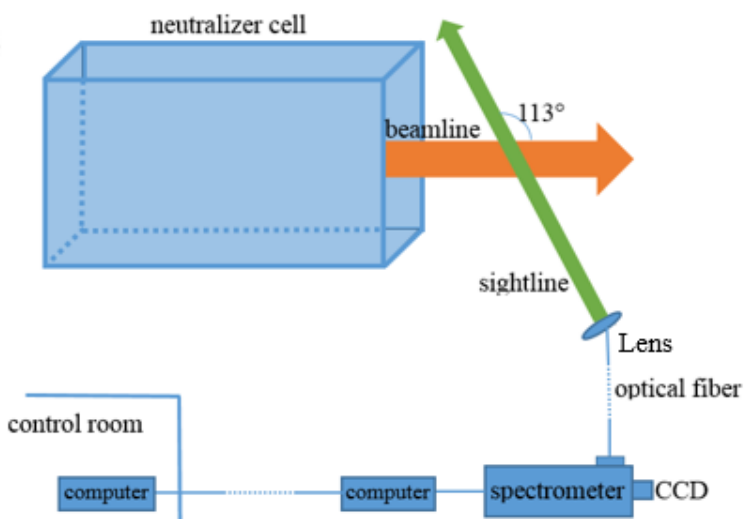

Figure 1. The sketch of (a) the neutral beam injector for EAST; (b) the DSS optical diagnostic system

\section{Calculation of Beam Components}

On the EAST-NBI test bed, the positive ion source adopts hydrogen as working gas and extracts three ionic components $\mathrm{H}^{+}, \mathrm{H}_{2}{ }^{+}$and $\mathrm{H}_{3}{ }^{+}$with the same kinetic energy E. Undergo the process of dissociation, ionization, combination with the gas target molecules in the neutralizer cell, the molecular ions dissociate into half-energy and third-energy atoms along with the full-energy atoms, finally 14 sorts of particles are formed in three groups (Khachan \& Collis, 2001; Wei, Hu, Liang, \& Xie, 2013; Wei, Hu, Xie, \& Liang, 2013; Yoo, Yang, \& Hwang, 1999):

$$
\begin{aligned}
& \mathrm{H}^{+}(\mathrm{E}) \text { ions: } \mathrm{H}^{+}(\mathrm{E}), \mathrm{H}(\mathrm{E}), \mathrm{H}^{-}(\mathrm{E}) ; \\
& \mathrm{H}_{2}{ }^{+}(\mathrm{E}) \text { ions: } \mathrm{H}^{+}(\mathrm{E} / 2), \mathrm{H}(\mathrm{E} / 2), \mathrm{H}^{-}(\mathrm{E} / 2), \mathrm{H}_{2}{ }^{+}(\mathrm{E}), \mathrm{H}_{2}(\mathrm{E}) ; \\
& \mathrm{H}_{3}{ }^{+}(\mathrm{E}) \text { ions: } \mathrm{H}^{+}(\mathrm{E} / 3), \mathrm{H}(\mathrm{E} / 3), \mathrm{H}^{-}(\mathrm{E} / 3), \mathrm{H}_{2}{ }^{+}(2 \mathrm{E} / 3), \mathrm{H}_{2}(2 \mathrm{E} / 3), \mathrm{H}_{3}{ }^{+}(\mathrm{E}) .
\end{aligned}
$$

Each particle fraction can be calculated as a function of the gas-target thickness for various particle energies, based on the law of conservation of particle number in each group, the evolution equation (Kim \& Haselton, 1979; Williams, Geddes, \& Gilbody, 1982; Yoo, Yang, \& Hwang, 2000) (1) is:

$$
\frac{d n^{j}}{d \pi}=\sum_{i \neq j}\left(c_{i j} n^{i}-c_{j i} n^{j}\right)
$$

Where $n$ denotes the particle relative number in each group, $c_{i j}$ denotes the reaction cross-section that the particle changes from $i$ to $j, \pi=\int n_{g} d l$ is the gas target thickness along the beam-line, $n_{g}$ is the gas density in the neutralizer cell. The bigger the gas target thickness, the higher efficiency of neutralization. At the best gas target thickness that $\pi$ is enough high, all particles will reach dynamic equilibrium under the complete dissociation of extracted molecular ions and the equilibrium between ionization and charge-exchange. Then, Equation (1) has been used to calculate the particle relative proportion in every group, for example $\mathrm{H}^{+}(\mathrm{E})$ fraction is as Equation (2) 


$$
f_{1}^{+}(E)=\frac{n^{H_{1}^{+}(E)}}{n_{0}^{H_{1}^{+}(E)}}=\frac{n^{H_{1}^{+}(E)}}{n^{H_{1}^{+}(E)}+n^{H_{1}^{0}(E)}+n^{H_{1}^{*}(E)}}
$$

In the experiment, since we measure the $\mathrm{H} \alpha$ lines which is collected between neutralizer and bending magnet where the background gas pressure is low, the residual gas density and the beam species density along the beam-line are constant. And electron density is lower than the gas density, so collisional de-excitation is low compared to the radiative decay rate, so we can use the corona model (Polosatkin, 2013; Umeda et al., 2006) to calculate the population of the excited states in the neutral atomic beam, where collisional excitation of a level is balanced by the radiative decay (Uhlemann et al., 1993). The $\mathrm{H} \alpha$ lines are emitted from the $n=3$ to $n=2$ state of the excited hydrogen atoms by collisions of the beam with the background gas, and the processes of transition have $3 \mathrm{~s}-2 \mathrm{p}, 3 \mathrm{p}-2 \mathrm{~s}$ and $3 \mathrm{~d}-2 \mathrm{p}$ under selection rule. The emissivity is defined as Equation (3):

$$
\frac{d N\left(H^{*}\right)}{d t}=\sigma_{i} v_{i} n_{i} n_{H_{2}}
$$

$v_{i}, n_{i}$ show the velocity and density of the particle, $\sigma_{i}$ is the corresponding excitation cross section factor, $n_{H 2}$ is the density of the background gas. Finally, based on the previous analysis, the ratio of the beam species is derived as Equation (4):

$$
\eta_{H^{+}}: \eta_{H_{2}^{+}}: \eta_{H_{3}^{+}}=\frac{I_{1}}{\sigma_{1}}: \frac{I_{2}}{\sigma_{2}}: \frac{I_{3}}{\sigma_{3}}
$$

Where $I_{1}, I_{2}, I_{3}$ denote the light intensity of each peak respectively, represented by the peak area. The expressions $\left(\sigma_{1}, \sigma_{2}, \sigma_{3}\right)$ are as Equations (5), (6), and (7):

$$
\begin{gathered}
\sigma_{1}=\sigma_{1}^{0} f_{1}^{0}(E)+\sigma_{1}^{+} f_{1}^{+}(E) \\
\sigma_{2}=\sigma_{1}^{0}\left(\frac{E}{2}\right) f_{1}^{0}\left(\frac{E}{2}\right)+\sigma_{1}^{+}\left(\frac{E}{2}\right) f_{1}^{+}\left(\frac{E}{2}\right)+\sigma_{2}^{0}(E) f_{2}^{0}(E)+\sigma_{2}^{+}(E) f_{2}^{+}(E) \\
\sigma_{3}=\sigma_{1}^{0}\left(\frac{E}{3}\right) f_{1}^{0}\left(\frac{E}{3}\right)+\sigma_{1}^{+}\left(\frac{E}{3}\right) f_{1}^{+}\left(\frac{E}{3}\right)+\sigma_{2}^{0}\left(\frac{2 E}{3}\right) f_{2}^{0}\left(\frac{2 E}{3}\right)+\sigma_{2}^{+}\left(\frac{2 E}{3}\right) f_{2}^{+}\left(\frac{2 E}{3}\right)+\sigma_{3}^{+}(e) f_{3}^{+}(E)
\end{gathered}
$$

In the above expression, $\sigma_{b}^{a}$ denotes the excitation cross-section of the corresponding particle $(\mathrm{a}=0,1, \mathrm{~b}=1,2,3)$, which is the function of beam particle energy.

\section{Experiment Results}

\subsection{DSS Spectrum}

A typical DSS spectrum of the $\mathrm{H} \alpha$ lines emitted by $46 \mathrm{keV}$ neutral beam for the shot 30117 is shown in Figure 2. The most intense unshifted peak is emitted by the background gas coming from the arc chamber and the neutralizer cell. The smallest peak is produced by low energy hydrogen atoms which result from the dissociation of $\mathrm{H}_{2} \mathrm{O}^{+}$ions. The three most shifted peaks are emitted by excited hydrogen atoms coming from the $\mathrm{H}^{+}, \mathrm{H}_{2}{ }^{+}$and $\mathrm{H}_{3}{ }^{+}$ions, corresponding to energy of $\mathrm{E}, \mathrm{E} / 2$, and $\mathrm{E} / 3$, where $\mathrm{E}$ is the accelerated energy. The baseline signal peak is also shown in Figure 2. Generally the hypothesis is that each peak is composed of spectral lines emitted by the same velocity particles, all peaks can be separated. Figure 2 shows the spectrum of blue shift acquired from the lens. It is assumed that the spectral lines meet Gaussian distribution. Six Gaussian peaks are fitted to the DSS data and the fitting curves are in good agreement with the experimental data.

\subsection{Analysis of Beam Components}

Figure 3 shows the species fraction as a function of the arc power for the acceleration voltage of $42 \mathrm{kV}$. The $\mathrm{H}^{+}$ fraction and $\mathrm{H}_{2}{ }^{+}$fraction increase, whereas the fraction of $\mathrm{H}_{3}{ }^{+}$decreases with the arc power increasing. It is mainly because more energetic electrons produced in the plasma generator make more molecular ions dissociate to atomic ions as the arc power rises (Burrell et al., 1980). The results suggest that, the arc power should be increased in order to extract an ion beam in which the $\mathrm{H}^{+}$ions are dominant in the experiment. 


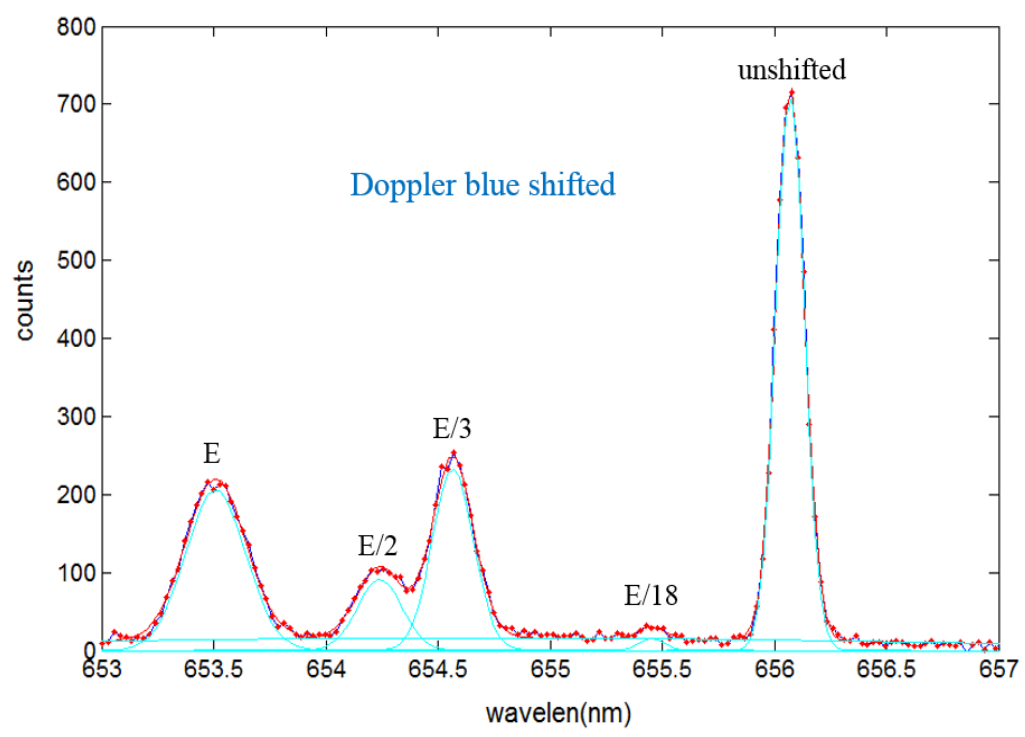

Figure 2. Spectrum of the $\mathrm{H} \alpha$ lines emitted by a $46 \mathrm{keV}$ beam

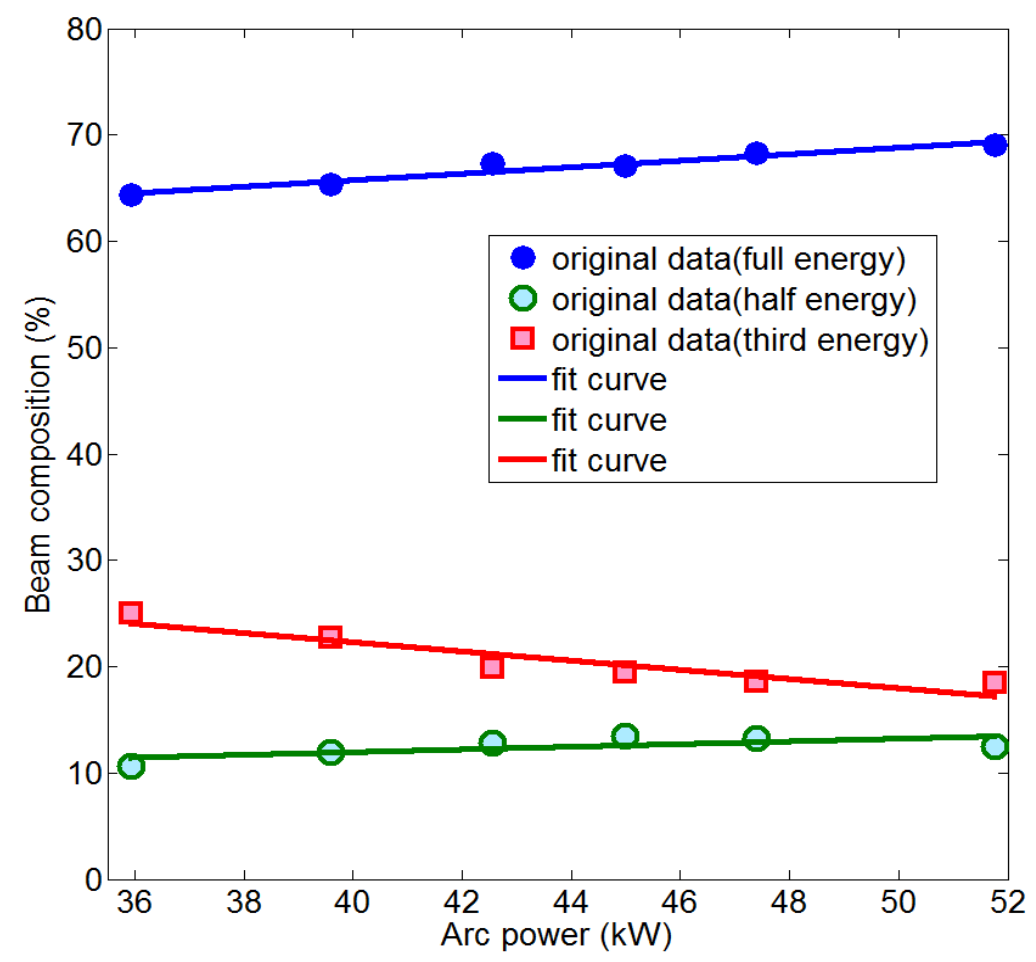

Figure 3. Beam species as a function of the arc power with the acceleration voltage of $42 \mathrm{kV}$

The variation of the beam compositions as a function of the beam energy is shown in Figure 4, where the perveance (defined by the ratio of the beam current to the extraction voltage) is held fixed at $2.4 \mu \mathrm{P}$. As the beam energy increases, the fractions of $\mathrm{H}^{+}$and $\mathrm{H}_{2}{ }^{+}$ions have a steady increase while the $\mathrm{H}_{3}{ }^{+}$fraction decreases. The proton ratio is about $75 \%$ when the beam energy is $70 \mathrm{keV}$. The main reasons perhaps are: 1 ) when the perveance is held fixed at $2.4 \mu \mathrm{P}$, this increase of beam energy is equivalent to increasing beam current. It means that the higher the densities of the primary ions and electrons are, so the more fraction of $\mathrm{H}^{+}$ions increases due to more collisional of molecular ions; 2) maybe there are more collisional dissociations of molecular ions in the extraction process as the accelerating voltage increases. 


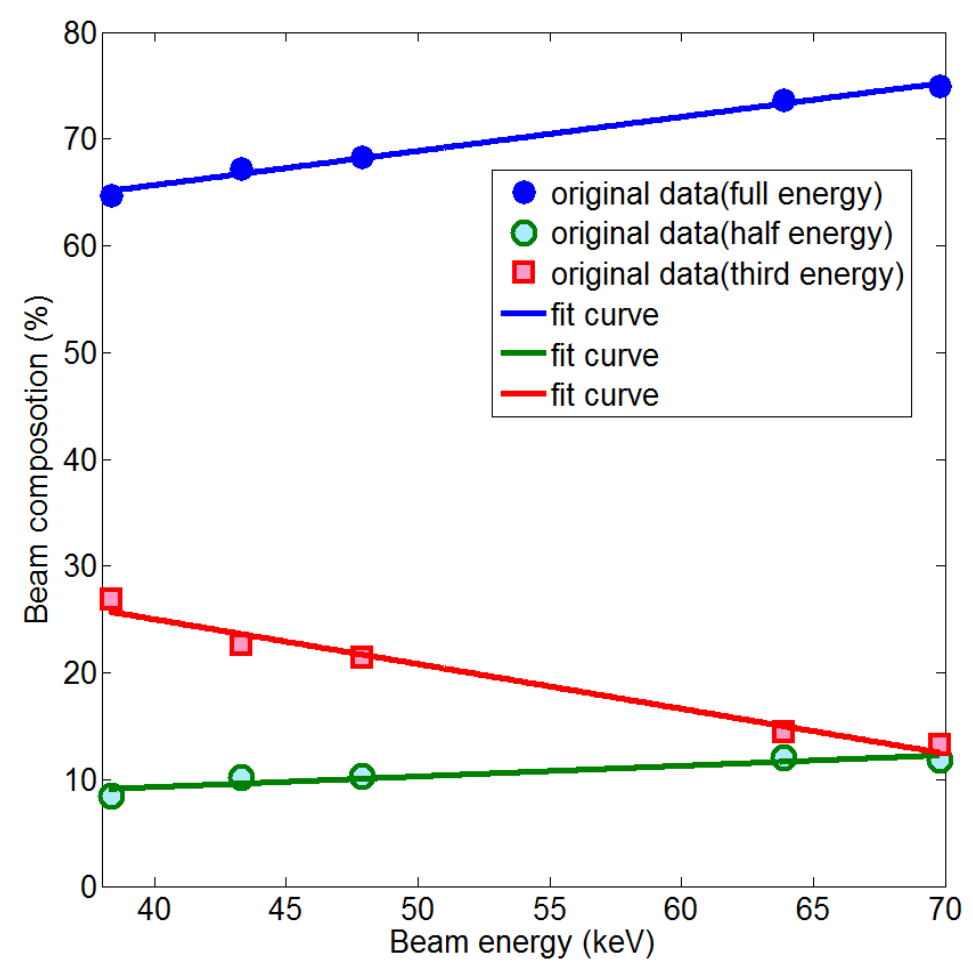

Figure 4. Beam species as a function of the beam energy with the perveance held $2.4 \mu \mathrm{P}$

On the EAST NBI testbed, we have conducted a number of experiments of the perveance which is from 2.4 to 3.1 with different parameters. In Figure 5, when the beam energy retain constant, the full energy species and half energy species increase, while the third energy species decrease as the perveance goes up. The maximum increasing rate achieves about $10 \%$. So we may increase the optimal perveance corresponding to minimum beam divergence within a certain range appropriately, to improve the proton ratio in the future experiment.

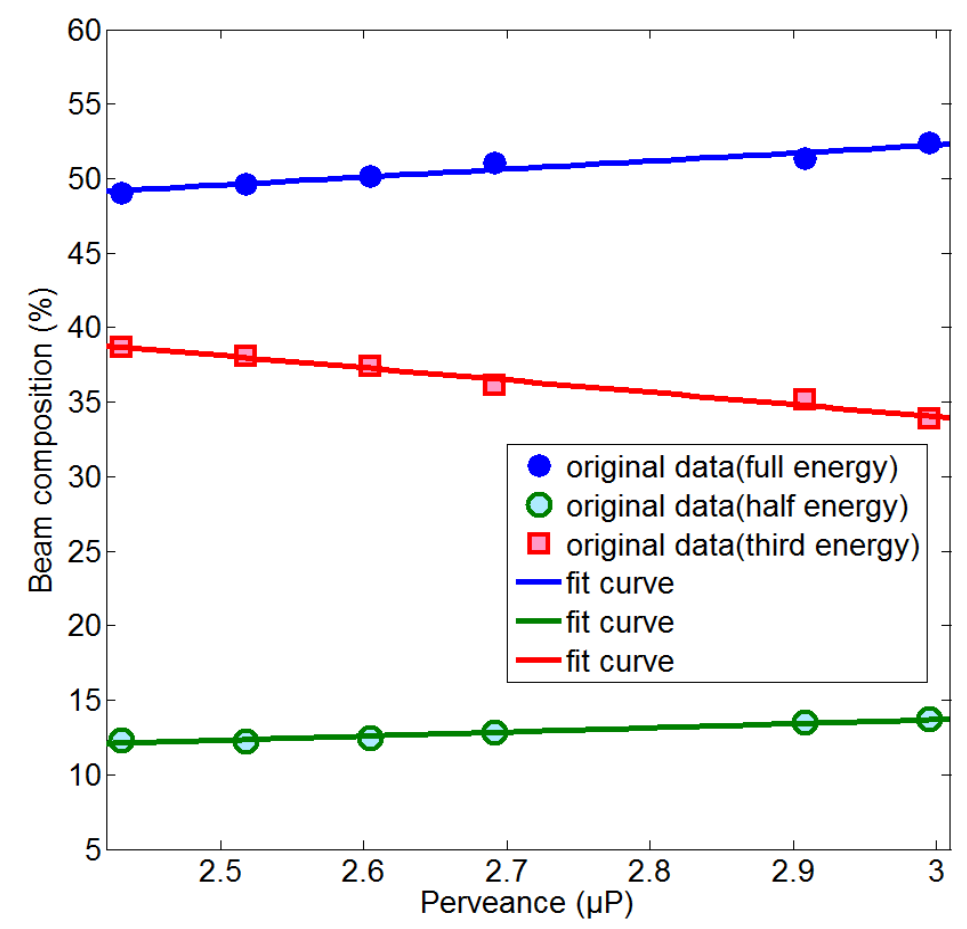

Figure 5. Beam species as a function of the perveance with the beam energy held constant 
Figure 3, 4, and 5 show essentially the beam species variations with source parameters, such as the filament voltage, the arc voltage, and the arc current, but we can also change the beam compositions by tuning the gas flow rate in the ion source. Figure 6 shows the beam compositions vary with the gas flow rate for the acceleration voltage of $40 \mathrm{kV}$ at the same arc power. We find that the full energy species fraction is not very sensitive to the gas flow rate within the range of experimental measurement. The fraction of $\mathrm{H}_{2}{ }^{+}$ions decreases and the fraction of $\mathrm{H}_{3}{ }^{+}$ions increases. When the gas pressure is larger than the required operation value in the plasma generator, $\mathrm{H}_{2}{ }^{+}$ions convert to $\mathrm{H}_{3}{ }^{+}$ions (Burrell et al., 1980). The process for producing $\mathrm{H}_{3}{ }^{+}$in the ion source is $\mathrm{H}_{2}{ }^{+}+\mathrm{H}_{2} \rightarrow \mathrm{H}_{3}{ }^{+}+\mathrm{H}$. So the gas flow rate should be set as an appropriate value, it is expected that both the arc chamber produces enough high density of plasma and the $\mathrm{H}_{3}{ }^{+}$fraction is a smaller amount.

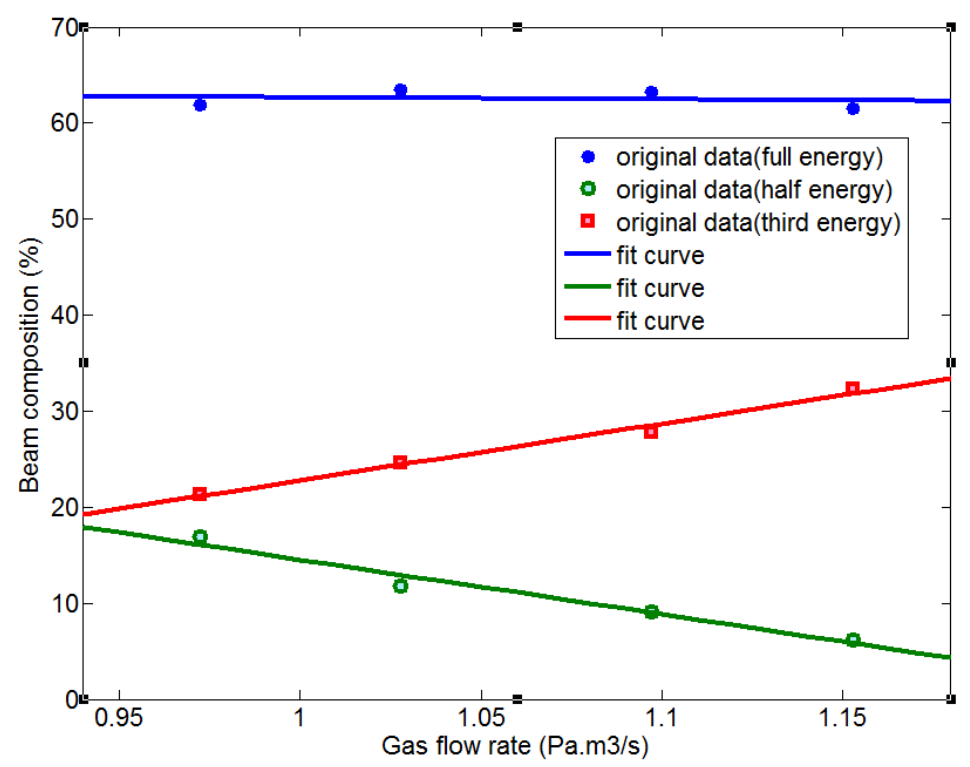

Figure 6. Beam species as a function of the gas flow rate for the acceleration voltage of $40 \mathrm{kV}$

\section{Conclusion}

The main purpose of the testing experiment is to diagnose the beam species characteristics and optimize the high-current ion source performance by tuning the operational parameters which include the arc power, the beam energy, the perveance, the gas flow rate. The experimental results obtained by the Doppler Shift Spectroscopy (DSS) optical diagnostic system show that the beam species fractions remain almost the same tendency as the arc power, the arc current, the beam energy and the perveance increase: the fractions of $\mathrm{H}^{+}$and $\mathrm{H}_{2}{ }^{+}$ions increase, of $\mathrm{H}_{3}{ }^{+}$ions decreases. The fraction of $\mathrm{H}_{3}{ }^{+}$ions can be minimized by tuning the gas flow rate.

Simultaneously, we will further take into account of optimizing the magnet configuration in order to tailor the electron energy in the extraction region to influence the ion species yield, and the optimizer of Gaussian fitting program and the cross section fitting to improve the accuracy of the calculation results. Based on the testing results, the optimal parameters are sorted out to a database document which provides the reference of extracting the high proton ratio neutral beam for EAST.

\section{Acknowledgments}

The authors would like to thank the other members of the EAST-NBI Group in Institute of Plasma Physics, Chinese Academy of Science. This work was supported by the National Magnetic Confinement Fusion Science Program of China (Contract No. 2013GB101000) and the National Natural Science Foundation of China (No. 1157240, 1150224).

\section{References}

Burrell, C. F., Cooper, W. S., Smith, R. R., \& Steele, W. F. (1980). Doppler shift spectroscopy of powerful neutral beams. Review of Scientific Instruments, 51(11), 1451. http://dx.doi.org/10.1063/1.1136124

Hu, C. D., \& Team, N. (2012). Preliminary Results of Ion Beam Extraction Tests on EAST Neutral Beam Injector. Plasma Science \& Technology, 14(10), 871-873. http://dx.doi.org/10.1088/1009-0630/14/10/03 
Hu, C. D., Xie, Y. H., \& Team, N. (2012). The Development of a Megawatt-Level High Current Ion Source. Plasma Science \& Technology, 14(1), 75-77. http://dx.doi.org/Doi 10.1088/1009-0630/14/1/16

Jeong, S. H., Chang, D. H., Kim, T. S., In, S. R., Lee, K. W., Jin, J. T., . . Hanada, M. (2012). First neutral beam injection experiments on KSTAR tokamak. Review of Scientific Instruments, 82(2). http://dx.doi.org/ $10.1063 / 1.3660254$

Kamperschroer, J. H., Kugel, H. W., Reale, M. A., Hayes, S. L., Johnson, G. A., Lowrance, J. L., ... Zucchino, P. M. (1987). Multiple track Doppler-shift spectroscopy system for TFTR neutral beam injectors. Review of Scientific Instruments, 58(8), 1362. http://dx.doi.org/10.1063/1.1139416

Khachan, J., \& Collis, S. (2001). Measurements of ion energy distributions by Doppler shift spectroscopy in an inertial-electrostatic confinement device. Physics of Plasmas, 8(4), 1299. http://dx.doi.org/10.1063/1.1349875

Kim, J., \& Haselton, H. H. (1979). Analysis of particle species evolution in neutral-beam injection lines. Journal of Applied Physics, 50(6), 3802. http://dx.doi.org/10.1063/1.326504

Liang, L. Z., Hu, C. D., Xie, Y. L., Guo, Q., \& Xie, Y. H. (2011). Calculation of Beam Intensity Distribution for the Neutral Beam Injection in EAST. Plasma Science \& Technology, 13(4), 502-505. http://dx.doi.org/10. 1088/1009-0630/13/4/22

Liang, L. Z., Hu, C. D., Xie, Y. L., Xie, Y. H., \& Nbi-Team. (2010). Modeling process of the neutral beam re-ionization loss. Chinese Physics C, 34(7), 972-977.

Polosatkin, S. (2013). Effect of sublevel population mixing on the interpretation of doppler-shift spectroscopy measurements of neutral beam content. Journal of Instrumentation, 8(05), P05007-P05007. http://dx.doi.org/10.1088/1748-0221/8/05/p05007

Thomas, D. M., Grierson, B. A., Burgos, J. M. M., \& Van Zeeland, M. A. (2012). Determination of neutral beam energy fractions from collisional radiative measurements on DIII-D. Review of Scientific Instruments, 83(10). http://dx.doi.org/10.1063/1.4733614

Tsumori, K., Takeiri, Y., Nagaoka, K., Ikeda, K., Osakabe, M., Oka, Y., ... Asano, E. (2006). High power neutral beam injection in LHD. Plasma Science \& Technology, 8(1), 24-27. http://dx.doi.org/10.1088/1009-0630/8/1/6

Uhlemann, R., Hemsworth, R. S., Wang, G., \& Euringer, H. (1993). Hydrogen and deuterium ion species mix and injected neutral beam power fractions of the TEXTOR-PINIs for 20-60 kV determined by Doppler shift spectroscopy. Review of Scientific Instruments, 64(4), 974. http://dx.doi.org/10.1063/1.1144100

Umeda, N., Ikeda, Y., Hanada, M., Inoue, T., Kawai, M., Kazawa, M., . . Ohga, T. (2006). Beam deflection by plasma grid filter current in the negative-ion source for JT-60U neutral beam injection system. Review of Scientific Instruments, 77(3). http://dx.doi.org/10.1063/1.2170088

Wei, J. L., Hu, C. D., Liang, L. Z., \& Xie, Y. L. (2013). Gas Flow in the Neutralization Region of EAST Neutral Beam Injector. Journal of Fusion Energy, 32(2), 196-202. http://dx.doi.org/10.1007/s10894-012-9548-y

Wei, J. L., Hu, C. D., Xie, Y. L., \& Liang, L. Z. (2013). Optimization of the gas flow in the neutralization region of EAST neutral beam injector. Fusion Engineering and Design, 88(12), 3176-3179. http://dx.doi.org/10. 1016/j.fusengdes.2013.09.014

Williams, I. D., Geddes, J., \& Gilbody, H. B. (1982). Balmer $\alpha$ emission in collisions of $\mathrm{H}, \mathrm{H}+, \mathrm{H} 2+$ and H3+with H2. Journal of Physics B: Atomic and Molecular Physics, 15(9), 1377-1389. http://dx.doi.org/10. 1088/0022-3700/15/9/014

Yoo, S. J., Yang, H. L., \& Hwang, S. M. (1999). Doppler shift spectroscopy for the measurement of angular divergence and ion species of ion beam. Journal of the Korean Physical Society, 35(4), 315-320.

Yoo, S. J., Yang, H. L., \& Hwang, S. M. (2000). Measurement of angular divergence and ion species ratios of an rf-driven multicusp ion source for diagnostic neutral beam by Doppler shift spectroscopy. Review of Scientific Instruments, 71(3), 1421. http://dx.doi.org/10.1063/1.1150473

\section{Copyrights}

Copyright for this article is retained by the author(s), with first publication rights granted to the journal.

This is an open-access article distributed under the terms and conditions of the Creative Commons Attribution license (http://creativecommons.org/licenses/by/3.0/). 